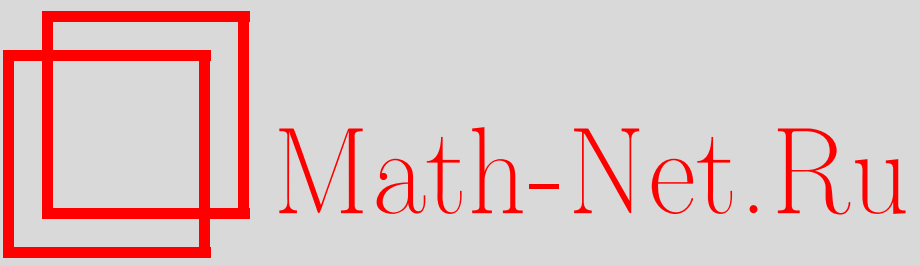

Н. Н. Ганиходжаев, Ф. М. Мухамедов, Эргодические свойства квантовых квадратичных стохастических процессов, определенных на алгебрах фон Неймана, УМН, 1998, том 53, выпуск 6, 243-244

DOI: https://doi.org/10.4213/rm97

Использование Общероссийского математического портала Math-Net.Ru подразумевает, что вы прочитали и согласны с пользовательским соглашением

http: //www.mathnet.ru/rus/agreement

Параметры загрузки:

IP : 54.209 .52 .79

26 апреля 2023 г., 13:48:26 


\title{
ЭРГОДИЧЕСКИЕ СВОЙСТВА КВАНТОВЫХ КВАДРАТИЧНЫХ СТОХАСТИЧЕСКИХ ПРОЦЕССОВ, ОПРЕДЕЛЕННЫХ НА АЛГЕБРАХ ФОН НЕЙМАНА
}

\author{
Н. Н. ГАНИХОДЖАЕВ,. М. МУХАМЕДОВ
}

В работах [1], [2] были определены квадратичные стохастические процессы, которые естественным образом возникают при изучении некоторых моделей со взаимодействиями. В [3] были введены квантовые квадратичные стохастические процессы на алгебрах фон Неймана, которые в частном случае совпадают с квадратичными стохастическими процессами.

В предлагаемой работе изучаются некоторые эргодические свойства квантовых квадратичных стохастических процессов.

Пусть $M$ - алгебра фон Неймана. Через $S$ и $S^{2}$ обозначим совокупности всех нормальных состояний на $M$ и $M \otimes M$ соответственно. Пусть далее $\left\{P^{s, t}: M \rightarrow M \otimes M, s, t \in \mathbb{R}_{+}, t-s \geqslant 1\right\}$ - семейство линейных операторов и $U: M \otimes M \rightarrow M \otimes M$ - оператор, которьй для элементов вида $x \otimes y$ определяется как $U(x \otimes y)=y \otimes x$ и далее продолжается на все $M \otimes M$ по линейности с сохранением нормы.

ОПРЕДЕЛЕниЕ 1. Будем говорить, что семейство линейных операторов $\left\{P^{s, t}, s, t \in \mathbb{R}_{+}\right\}$ определяет көантовый квадратичный стохастический прочесс (к.к.с.п.), если каждый оператор $P^{s, t}$ ультраслабо непрерьвен и при этом выполняются следующие соотношения:

(i) $P^{s, t} 1_{M}=1_{M \otimes M}$ для всех $s, t \in \mathbb{R}_{+}$, где $1_{M}$ и $1_{M \otimes M}$ - единицы алгебр $M$ и $M \times M$ соответственно;

(ii) $P^{s, t}\left(M_{+}\right) \subset(M \otimes M)_{+}$для всех $s, t \in \mathbb{R}_{+}$;

(iii) $P^{s, t}(M)$ симметрично, т.е. $P^{s, t}(M) \subseteq\{x \in M \otimes M: U x=x\}$ для всех $s, t \in \mathbb{R}_{+}$;

(iv) (аналог уравнения Колмогорова-Чепмена) для начального состояния $\omega_{0} \in S$ и произвольных $s, \tau, t \in \mathbb{R}_{+}$таких, что $\tau-s \geqslant 1, t-\tau \geqslant 1$, либо

(iv) ${ }_{\mathrm{A}} P^{s, t} x=\left(P^{s, \tau} \circ \omega_{\tau}\right)\left(P^{\tau, t} x\right), x \in M$, либо

(iv) $)_{\mathrm{B}} P^{s, t} x=\omega_{s} \otimes \omega_{s}\left(P^{s, \tau} \circ P^{s, \tau}\right)\left(P^{\tau, t} x\right), x \in M$, где $\omega_{\tau}(x)=\omega_{0} \otimes \omega_{0}\left(P^{0, \tau} x\right), x \in M$, и по определению полагается:

$$
\begin{aligned}
\left(P^{s, t} \circ \omega_{t}\right)(x \otimes y) & :=\omega_{t}(y) P^{s, t} x, \\
\omega_{t} \otimes \omega_{t}(x \otimes y \circ a \otimes b) & :=\omega_{t}(y) \omega_{t}(b) x \otimes a, \\
\left(P^{s, t} \circ P^{s, t}\right)(x \otimes y) & :=P^{s, t} x \circ P^{s, t} y .
\end{aligned}
$$

Если к.к.с.п. удовлетворяет равенству (iv) А или (iv) В, то будем говорить, что к.к.с.п. имеет mun (A) или тип (В) соответственно.

Будем говорить, что к.к.с.п. $\left\{P^{s, t}\right\}$ однороден, если $P^{s, t}$ зависит только от $t-s$, для любых $s$ и $t$ таких, что $t-s \geqslant 1$.

Множество всех непрерьвных (ультраслабо непрерывных) функционалов на $M$ обозначим через $M^{*}\left(M_{*}\right)$. Через $P_{*}^{s, t}$ обозначим оператор, действующий из $(M \otimes M) *$ в $M_{*}$ и определенньй следующим образом: $P_{*}^{s, t}(\varphi)(x)=\varphi\left(P^{s, t} x\right), \varphi \in(M \otimes M)_{*}, x \in M$. Норму на $M^{*}$ будем обозначать $\|\cdot\|_{1}$

ОПРЕДЕЛЕнИЕ 2. Будем говорить, что к.к.с.п. $\left\{P^{s, t}\right\}$ удовлетворяет әргодическому принu uny, если для любых $\varphi, \psi \in S^{2}$ и $s \in \mathbb{R}_{+}$имеет место $\lim _{t \rightarrow \infty}\left\|P_{*}^{s, t} \varphi-P_{*}^{s, t} \psi\right\|_{1}=0$. 
Теорема 1. Пусть $M-$ алгебра фон Неймана $u\left\{P^{s, t}\right\}-$ к.к.c.n. на M. Для к.к.c.n. $\left\{P^{s, t}\right\}$ выполнен эргодический принцип тогда и только тогда, когда найдутся такое всюду плотное множество $\mathfrak{F} \subset S^{2}$ и действительное число $\lambda \in[0,1)$, что для каждой пары $\varphi, \psi \in \mathfrak{F}$ и $s \in \mathbb{R}_{+}$будет выполнено неравенство

хотя бъь для одного $t=t(s, \varphi, \psi)$.

$$
\left\|P_{*}^{s, t} \varphi-P_{*}^{s, t} \psi\right\|_{1} \leqslant \lambda\|\varphi-\psi\|_{1}
$$

Заметим, что аналогичная теорема была доказана в [4] для однородного марковского процесса с дискретным временем, определенного на упорядоченном пространстве.

ОПредЕЛЕНИЕ 3. Будем говорить, что к.к.с.п. $\left\{P^{s, t}\right\}$ на $M$ удовлетворяет условию регулярности (әкспоненииальной), если существует состояние $\mu_{1} \in S$ такое, что для любого $\varphi \in S^{2}$ и $s \in \mathbb{R}_{+}\left\|P_{*}^{s, t} \varphi-\mu_{1}\right\|_{1} \rightarrow 0$ при $t \rightarrow \infty\left(\left\|P_{*}^{s, t} \varphi-\mu_{1}\right\|_{1} \leqslant d e^{-b t}\right.$ для любых $\varphi \in S^{2}$ и $t \geqslant t_{0}$, где $d, b>0$ ).

Будем говорить, что к.к.с.п. $\left\{P^{s, t}\right\}$ удовлетворяет услов ию $\left(\mathrm{A}_{1}\right)$ на $\mathfrak{R} \subset S^{2}$, если существуют число $\lambda \in(0,1]$ и семейство состояний $\left\{\mu_{s}^{t}: s, t \in \mathbb{R}_{+}, t-s \geqslant 1\right\} \subset S$ такие, что для любых $\varphi \in \Re, s \in \mathbb{R}_{+}$найдется число $t_{0}=t_{0}(\varphi, s)$, для которого $\varphi \circ P^{s, t} \geqslant \lambda \mu_{s}^{t}$ при всех $t \geqslant t_{0}$.

Теорема 2. Пусть к.к.c.n. $\left\{P^{s, t}\right\}$ на алгебре фон Неймана $M$ удовлетворяет условию $\left(\mathrm{A}_{1}\right)$ на множестве $\mathfrak{R}$, выпуклая оболочка которого $\sigma\left((M \otimes M)_{*}, M \otimes M\right)$-слабо плотна в $S^{2}$. Тогда к.к.c.n. $\left\{P^{s, t}\right\}$ удовлетворяет әргодическому принципу.

Доказательство получается непосредственной проверкой условия теоремы 1.

Заметим, что теорема 2 обобщает резултаты работы [5].

Лемма 1. Пусть $\left\{P^{s, t}\right\}-$ однородный к.к.c.n. на алгебре фон Неймана М. Тогда имеет место равенство $\omega_{2}=\omega_{t}$ при всех $t \geqslant 2$.

Из этой леммы и теоремы 1 можно получить следующие теоремы.

Теорема 3. Пусть $\left\{P^{s, t}\right\}-$ однородный к.к.c.n. на алгебре фон Неймана М. Квантовый квадратичньй стохастический прочесс $\left\{P^{s, t}\right\}$ удовлетворяет условию регулярности тогда и только тогда, когда найдутся такое всюду плотное множество $\mathfrak{F} \subset S^{2}$ и действительное число $\lambda \in[0,1)$, что для каждой пары $\varphi, \psi \in \mathfrak{F}$ будет выполнено неравенство

хотя бъц для одного $\tau=\tau(\varphi, \psi)$.

$$
\left\|P_{*}^{0, \tau} \varphi-P_{*}^{0, \tau} \psi\right\|_{1} \leqslant \lambda\|\varphi-\psi\|_{1}
$$

Теорема 4. Пусть $\left\{P^{s, t}\right\}$ - однородный к.к.c.n. на алгебре фон Неймана М. Квантовый квадратичный стохастический процесс $\left\{P^{s, t}\right\}$ удовлетворяет условию әкспоненциальной регулярности тогда и только тогда, когда найдутся такое всюду плотное множество $\mathfrak{F} \subset S^{2}$ и действительное число $\lambda \in[0,1)$, что при некотором $\tau \in \mathbb{R}_{+}$ выполнено неравенство

$$
\left\|P_{*}^{0, \tau} \varphi-P_{*}^{0, \tau} \psi\right\|_{1} \leqslant \lambda\|\varphi-\psi\|_{1} \quad \text { для } \quad \text { всех } \quad \varphi, \psi \in \mathfrak{F} .
$$

\section{СПИСОК ЛИТЕРАТУРЫ}

[1] Сарымсаков Т. А., Ганиходжаев Н. Н. // Докл. АН СССР. 1989. Т. 305. № 5. С. 1052 -1056. [2] Sarymsakov T. A., Ganikhodzhaev N. N. // J. Theor. Probab. 1990. V. 3. № 1. Р. 51-70. [3] Ганиходжаев Н. Н., Мухамедов Ф. М. // Докл. АН РУз. 1997. № 3. С. 13-16. [4] Сарымсаков Т.А., Зимаков Н. П. // Операторные алгебры и функционалшные пространства. Ташкент: Фан, 1988. С. 45-53. [5] Ганиходжаев Н. Н., Мухамедов Ф. М. // Узб. матем. журн. 1997. № 3. С. 8-20. 